\title{
Evaluación de la relación entre el habito y frecuencia del consumo de lácteos y el nivel de calcio presente en la orina de jóvenes universitarios de la ciudad de Armenia
}

Cindy Roldan Sánchez ${ }^{1}$, M.sc Paola Orozco Santos², Adriana María Zuluaga Monsalve , M.sc Juan Carlos Lucas Aguirre ${ }^{4}$

1 Estudiante Octavo Semestre. Programa de Enfermería. Integrante Grupo de Semillero Salud en Comunitaria. Corporación Universitaria Empresarial Alexander Von Humboldt.

2 Docente Programa de Enfermería. Grupo de Semillero Salud en Comunitaria. Corporación Universitaria Empresarial Alexander Von Humboldt.

3 Directora de Investigación. Corporación Universitaria Empresarial Alexander Von Humboldt.

4: Docente Programa de Enfermería. Grupo de Semillero Salud en Comunitaria. Corporación Universitaria Empresarial Alexander Von Humboldt.

* Correo electrónico: *Correo electrónico: juanlucas9@cue.edu.co

Fecha de Recepción: 2/2/2016

Fecha de Evaluación: 3/02/2016

Fecha de Solicitud de Correcciones: 26/2/2016

Fecha de Aceptación: 3/03/2016

\section{Resumen}

La presente investigación evaluó los estilos de vida de jóvenes universitarios, en este caso la relación entre el hábito y frecuencia del consumo de lácteos y nivel de calcio presente en la orina de jóvenes entre los 18 y 25 años de la ciudad de Armenia. Se realizó un estudio exploratorio descriptivo, en 98 jóvenes a los cuales se les aplico un instrumento de valoración donde se obtuvo información acerca de sus estilos de vida, consumo de lácteos, y se les práctico un examen de calcio en orina ocasional. Los resultados obtenidos arrojaron que toda la población objeto de estudio consumen productos lácteos, los hombres prefieren consumir más la leche con un $60 \%$ que las mujeres con un $51 \%$. No se encontró correlación entre el consumo de lácteos y la cantidad de calcio presente en la Orina dado que los valores dieron muy cercanos a cero $(-0,0288035-0,12558183)$. No se evidenció correlación entre el consumo, frecuencia y tipo de producto lácteo consumido vs. cantidad de calcio encontrada en la orina y la alteración de los niveles de calcio en orina que darían origen al padecimiento de hipercalciuria o hipocalciuria, por lo cual es necesario buscar y correlacionar otras fuentes de calcio que eviten los padecimientos anteriormente mencionados.

Palabras clave: Hipercalciuria, Hipocalciuria. Vitamina D. Osteoporosis. DMO.
Evaluation of the relationship between the habit and frequency of consumption of milk and calcium this level in urine of college students of the city of Armenia

\begin{abstract}
This research assessed the lifestyles of university students, in this case the relationship between the habit and frequency of consumption of dairy products and calcium levels in the urine of young people between 18 and 25 years of the city of Armenia. an exploratory descriptive study, in which 98 young people to apply them as a valuable tool where information was obtained about their lifestyles, consumption of dairy products was performed and performed a calcium scan occasional urine. The results showed that the entire study population consume dairy products, men prefer to consume more milk with $60 \%$ women $51 \%$. No correlation between dairy intake and the amount of calcium in the urine was found since the values given very close to zero $(-0.0288035$ to 0.12558183$)$. No correlation between consumption, frequency and type of dairy product consumed evidenced vs. amount of calcium found in urine and altered levels of calcium in urine that would give rise to the condition of hypercalciuria or hypocalciuria, so you need to search and correlate other sources of calcium to avoid the aforementioned conditions.
\end{abstract}

Keywords: Hypercalciuria. Hypocalciuria. Vitamin D. Osteoporosis, BMD.

\section{Introducción}

Osteoporosis significa, literalmente, "porosidad en los huesos". Esta enfermedad es consecuencia de alteraciones del remodelamiento con anormalidades de la estructura o funciones óseas (1). La osteoporosis es una enfermedad esquelética sistémica caracterizada por una baja masa ósea y deterioro de la microarquitectura del tejido óseo, con el consiguiente aumento de la fragilidad ósea y la susceptibilidad a la fractura que ocurre principalmente en las mujeres posmenopáusicas a medida que envejecen y en menor grado en los hombres mayores, la osteoporosis es típicamente una enfermedad sin síntomas hasta que ocurre una fractura en el tejido óseo (2). Hay numerosos factores relacionados con el riesgo de fracturas óseas. Los principales factores de riesgo son el sexo, la edad, la densidad mineral ósea (DMO), una historia personal de fracturas debido a la fragilidad, antecedentes familiares de primer grado con fractura debido a la fragilidad, deficiencia de calcio, deficiencia de vitamina $\mathrm{D}$ y bajo peso corporal (índice de masa corporal [IMC] menor a $\left.20 \mathrm{~kg} / \mathrm{m}^{2}\right)(3)$.

Según los conocimientos actuales, habría que recomendar una dieta equilibrada con abundancia de productos lácteos, limitar el consumo de proteína animal y por el contrario aumentar el consumo de productos vegetales para de esta manera contribuir al buen aporte de calcio en las personas y evitar futuros trastornos de salud relacionados con la osteoporosis. No conviene suprimir los lácteos de la dieta ya que son las principales fuentes de calcio, con la alternativa de poder usar los desnatados para aquellas personas que tengan que cuidar la ingesta de grasa, aunque estos últimos tienen el inconveniente de que pierden las vitaminas liposolubles especialmente la vitamina D. En las personas con intolerancia a la lactosa que tengan molestias tras la ingestión de leche, se recomienda el consumo de yogur y leches fermentadas $(4,5)$. En general, se ha determinado que la leche y los productos lácteos son la principal fuente de calcio en los países occidentales, con una contribución aproximada del 70\% del consumo total de calcio (6). El calcio específicamente tiene las siguientes funciones: constitución de fluidos y tejidos, regulación cardíaca, componente de los sistemas enzimáticos, conducción nerviosa, proliferación celular, estimulante de la secreción hormonal, contracción muscular, coagulación sanguínea y la más importante, el mantenimiento de la estructura y calidad de la masa ósea (7). 
La vitamina $\mathrm{D}$ se forma en la piel por exposición a los rayos ultravioletas, se encuentra en muy pocos alimentos y su función es favorecer la absorción de calcio a nivel intestinal. Para alcanzar buenos niveles de vitamina $\mathrm{D}$ la exposición solar debe ser corta, entre 15 y 20 minutos, y siempre fuera de los horarios pico de mayor radiación solar. Esta exposición debe repetirse 2 o 3 veces por semana (8). Dentro de las recomendaciones para mejorar la salud respecto a la osteoporosis se encuentra el consumo de productos lácteos. Sería suficiente si se consume entre 1 a 4 vasos de leche al día, un yogur y una porción de queso al día (9). La leche de vaca y derivados son las fuentes más ricas y con la más alta absorción de Calcio. Sin embargo, otros alimentos, como frijoles blancos, brócolis, col rizada y los pescados pequeños enteros, podrían ser utilizados en asociación con productos de mayor tenor/biodisponibilidad, a fin de alcanzar los niveles dietéticos adecuados de Calcio en individuos que no quieren o no pueden ingerir leche de vaca (10).

La Hipercalciuria es un trastorno metabólico que muestra una alta concentración de calcio en la orina. Se clasifica en dos subtipos, absortiva y renal. La absortiva responde a un aumento en la absorción de calcio a nivel intestinal, y como consecuencia, incrementa la excreción urinaria. La renal, implica una pérdida de calcio que se compensa con una mayor absorción intestinal. La excreción constante de cantidades elevadas de calcio a través de la orina, hace que el organismo quite calcio de los huesos para mantener constante la concentración en sangre. La osteoporosis es el debilitamiento de la masa ósea por falta de calcio (1), mientras la Hipocalciuria es la disminución de la cantidad de calcio contenido en la orina (11).

En el presente trabajo se realizó un estudio exploratorio descriptivo de 98 jóvenes, a los cuales se les aplicó un instrumento de valoración, donde se obtuvo información acerca de sus estilos de vida, consumo de lácteos, y se les realizo un examen de calcio en orina ocasional para determinar los niveles de hipercalciuria e hipocalciuria.

\section{Materiales y métodos}

Se realizó un estudio descriptivo - exploratorio, en jóvenes universitarios (hombres y mujeres) entre los 18 y 25 años de edad de la ciudad de Armenia, Quindío-Colombia, utilizando como criterios de inclusión: no presentar padecimiento de alguna enfermedad sistémica, que no estuvieran embarazadas ni lactando en el caso de las mujeres. Los criterios de exclusión fueron pacientes (mujeres) que presentaran uso de anticonceptivos prolongados para planificación, que padecieran de enfermedades relacionadas con el metabolismo en especial problemas relacionados con deficiencias de calcio y daños de la estructura ósea, que presentaran ingesta de Vitamina D y C, calcio, flúor y bifosfonatos, que tuviera una práctica deportiva mayor a 2 horas/día, que presentara un índice de masa corporal bajo $(<16)$, que tuviera un DMO menor o igual -3 desviaciones estándar en $\mathrm{Z}$ score. Los pacientes que fueron aceptados para someterse a dicha investigación firmaron un consentimiento informado y se hicieron participes de manera voluntaria.

Se obtuvo el tamaño de la muestra (98 individuos) con un margen de error del $10 \%$ y una confiabilidad del $95 \%$ teniendo en cuenta la prevalencia de osteopenia que es entre 45 y $49 \%$ (12).

A los individuos se les diligencio una ficha clínica para la obtención de los datos necesarios para una valoración nutricional. Los datos incluyeron la historia médica del investigado, antecedentes tanto personales, familiares, como farmacológicos, su historia dietética, se determinaron sus estilos de vida, se les realizó un examen físico que incluyó la toma del peso, la talla, índice de masa corporal (IMC), pliegues cutáneos, sus signos vitales, y valoración general de su estado de salud. Los pacientes acudieron al laboratorio correspondiente en ayuno de 12 horas. Para la toma de la densitometría ósea se informó a los investigados que no consumieran suplementos de calcio durante las 24 horas previas al examen. El examen de densitometría dual de $\mathrm{Rx}$ se tomó con un densitómetro marca HOLOGIC Explorer QDR, en el cual se midió la densidad de cuatro regiones: cadera total, cuello femoral, columna lumbar para medición de DMO y cuerpo completo con el fin de determinar la composición corporal (grasa corporal y masa muscular). El densitómetro daba unos parámetros de media dependiendo de la edad; para jóvenes (pediátrico) de 18 a 20 años la cual evaluaba la densidad mineral ósea y composición corporal TBHL (grasa total) y para adultos jóvenes (adulto) de 21 a 25 años evaluaba el Z score de columna y fémur, y composición corporal TBHL. Para las densitometría ósea de rayos X (DXA) se consideraron los valores DMO normales a las DE en $\mathrm{Z}$ score > -2, o reserva de masa ósea baja a una DE en Z score -2 a -2.9. Se les realizó exámenes de Calcio en orina, con el fin de evaluar marcadores de resorción ósea. Esta recolección fue necesaria para determinar la relación calcio de los jóvenes en estudio; se consideró hipercalciuria en cifras $\geq 17 \mathrm{mg} /$ dal. Se analizaron las variables del estudio en una base de datos de Epi-info ${ }^{\star}$.

\section{Resultados}

\section{Relación entre sexo y Z-core:}

La relación entre sexo y Z-score L1-L4 DXO, adulto y pediátrico, fue con un $\mathrm{r}^{2}=0,0254$ y r${ }^{2}=0,0835$ respectivamente, siendo esto estadísticamente no significativo. La relación entre sexo y Z-score, cuello de fémur DXO adulto y pediátrico, con un $\mathrm{r}^{2}=0,2028$ y r ${ }^{2}=0,0248$ respectivamente, siendo esto estadísticamente no significativo. Se evaluó la realización de ejercicio donde se encontró que 60 de los jóvenes universitarios si realizan ejercicio equivalente al $61 \%$ y que los jóvenes que no realizan ningún tipo de ejercicio ascienden al 39\%.

\section{Relación entre ejercicio y Z-core:}

La relación entre ejercicio y Z-score L1-L4 DXO adulto y pediátrico, fue con un $r^{2}=2 \times 10^{-5} y$ de $r^{2}=0,0019$ respectivamente, siendo esto estadísticamente no significativo. La relación entre ejercicio y $\mathrm{z}$-score cuello de fémur DXO adulto y pediátrico, con un $\mathrm{r}^{2}=0,12$ y de $r^{2}=0,0747$ respectivamente, para lo cual estadísticamente no fue significativo.

Índice de Masa Corporal (IMC):

Se encontró que 66 jóvenes universitarios se encuentran con un IMC normal $(68 \%)$ y el $25 \%$ se encuentra por encima del IMC normal y el $11 \%$ se clasificó por debajo del IMC normal. Con lo anterior, se estableció una Delgadez aceptable el 6\% de la población estudiada, Delgadez moderada el 2\%, Delgadez severa el 2\%, Infrapeso el $1 \%$, Obeso el $2 \%$, Obeso tipo I el $2 \%$, Obeso tipo II el $1 \%$, Preobeso el $10 \%$ y Sobrepeso el 6\%.

\section{Relación entre IMC y z-core:}

La relación entre IMC y z-score L1-L4 DXO adulto y pediátrico, fue de $r^{2}=0,02$ y r $r^{2}=0,0169$ respectivamente, siendo esto estadísticamente no significativo. La relación entre IMC y z-score del cuello de fémur DXO adulto y pediátrico, fue de $r^{2}=0,0631$ y $r^{2}=0,0004$ respectivamente, siendo esto estadísticamente no significativo. 


\section{Análisis de calcio en orina:}

Gráfico 1. Porcentaje de calcio en orina de la población universitaria. Fuente propia.

\section{CALCIO EN ORINA DE TODA LA POBLACION}

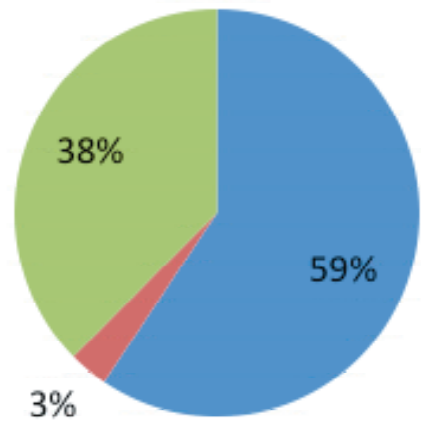

Normal

Hipocalciuria

Hipercalciuria

En el grafico 1 se observan los resultados de calcio en orina de la población evaluada, en general se encontró que el 59\% se encuentra entre los parámetros normales, el 38\% presentó hipercalciuria y el 3\% hipocalciuria.

\section{Análisis de consumo de lácteos:}

Gráfico 2. Porcentajes del consumo de lácteos en cada comida de la población universitaria.

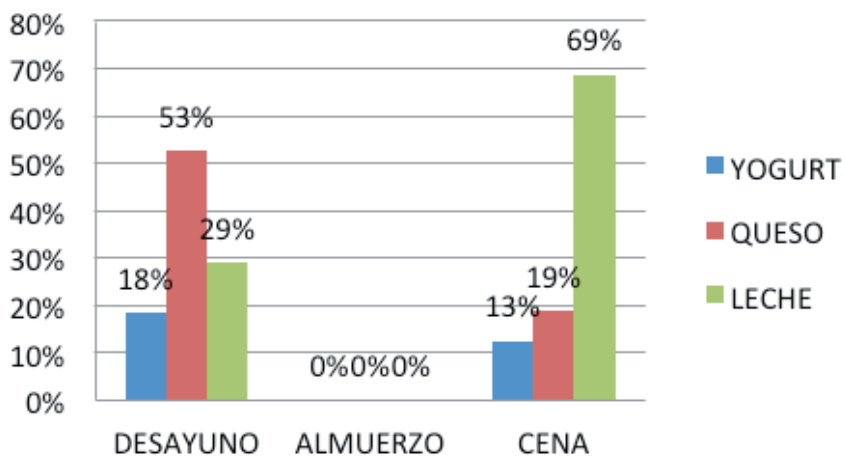

$\mathrm{Al}$ analizar los resultados del consumo de lácteos en cada comida de la población objeto, se encontró que en el desayuno el 53\% consumen queso, siendo el de mayor de consumo, seguido por la leche (29\%) y el yogurt $18 \%$. En el almuerzo en general no consumen ningún lácteo y para la comida un $69 \%$ consumen leche siendo el de mayor consumo, el $19 \%$ consumen queso y el $13 \%$ yogurt (ver gráfico 2 ).
Gráfico 3. Porcentajes de frecuencia del consumo de lácteos de la población universitaria.

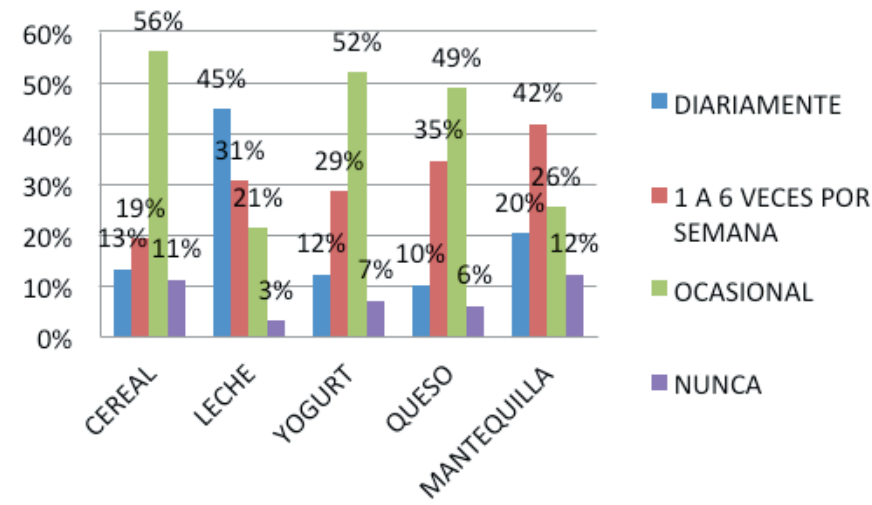

Por último al analizar los resultados de frecuencia de consumo de lácteos de la población estudiada, se encontró que el 56\% consume cereal ocasionalmente, el $19 \%$ una a seis veces por semana, el $13 \%$ diariamente y el $11 \%$ nunca consume cereal; para el consumo de leche se encontró que el $45 \%$ consume diariamente, el $31 \%$ una a seis veces por semana, el $21 \%$ ocasionalmente y el $3 \%$ nunca consume leche; para el consumo de yogurt se encontró que el $52 \%$ consume ocasionalmente, el $29 \%$ una a seis veces por semana, el $12 \%$ diariamente y el 7\% nunca consume yogurt; para el consumo de queso se encontró que el $49 \%$ consume ocasionalmente, el 35\% una a seis veces por semana, el $10 \%$ diariamente y el $6 \%$ nunca consume queso; y para el consumo de mantequilla se encontró que el $42 \%$ consume una a seis veces por semana, el $26 \%$ ocasionalmente, el $20 \%$ diariamente, y el $12 \%$ nunca consume mantequilla (Grafico 3).

\section{Análisis de coeficiente de relación de Pearson:}

Al buscar una posible correlación entre el consumo, frecuencia y el tipo de producto lácteo que consume la población en estudio vs. la cantidad de calcio encontrada en la orina, no se evidenció relación alguna puesto que dichos valores dieron muy cercanos a cero $(-0,0288035-0,12558183)$.

\section{Discusión}

El 38\% de la población estudiada presento hipercalciuria, lo que posiblemente se puede dar por la Nefropatía crónica, niveles muy altos de vitamina $\mathrm{D}$, escape de calcio desde los riñones hacia la orina lo cual provoca cálculos renales de calcio, sarcoidosis, ingesta alta de calcio, exceso en la producción de PTH (hiperparatiroidismo) y uso de diuréticos. Los niveles bajos de calcio en la orina podrían deberse a trastornos en los cuales el cuerpo no absorbe bien los nutrientes de los alimentos, trastornos en los cuales el riñón maneja el calcio de manera anormal, glándulas paratiroides en el cuello que no producen suficiente hormona paratiroidea (hiperparatiroidismo), uso de diuréticos tiazídicos y niveles muy bajos de vitamina $\mathrm{D}$ (13).

En cuanto a la correlación entre el consumo, frecuencia y el tipo de producto lácteo que consume la población en estudio frente a la cantidad de calcio encontrada en la orina de los pacientes, no se permite establecer una relación directa e indirecta entre el consumo, frecuencia y tipo de producto lácteo con la alteración de los niveles de calcio en orina que darían origen al padecimiento de hipercalciuria o hipocalciuria. Siendo la hipercalciuria la enfermedad de más relevancia y gravedad, se recomienda garantizar una adecuada ingesta de líquidos para la prevención de la sobresaturación de la orina, independientemente de la causa. Además, debe recomendarse una dieta baja en sodio, alta en potasio. Una dieta aumentada en potasio 
puede reducir la excreción urinaria de calcio. La situación de riesgo que supone una hipercalciuria asintomática exige únicamente la instauración de medidas dietéticas protectoras e incremento de la práctica de ejercicio físico, evitando crear conciencia de enfermedad. La dieta debe ajustarse a las recomendaciones diarias, la ingesta de proteínas, calcio y sal. Debe suspenderse el aporte de suplementos de vitamina $C$ y vitamina $D$ para evitar hipervitaminosis y de esta manera otras hipercalciurias normocalcemicas. Los cítricos (naranja, limón) tienen un alto contenido en citrato, que es el principal inhibidor de la cristalización por lo cual es recomendable evitar en cierta medida los citratos en primer lugar, en segundo lugar la excreción urinaria de potasio disminuye la calciuria. Muchas frutas son además ricas en potasio (sandía, melón, naranja, mandarina, uvas, plátano) a diferencia de los cereales integrales y el pescado azul (atún, sardinas, salmón, etc.) que tienen la ventaja de aportar factores protectores de la formación de cálculos (14).

En énfasis el total de la población consume productos lácteos siendo así el de mayor preferencia el consumo de leche, dentro de la frecuencia se evidenció un alto porcentaje en el consumo de lácteos una vez al día con un 48\%, el 35\% dos veces al día, el 16\% más de tres veces al día y el $1 \%$ no consume. La leche la consumen diariamente en un alto porcentaje (45\%) y para los demás lácteos lo consumen ocasionalmente como el cereal (56\%), yogurt (52\%) y el queso (49\%); la mantequilla la consumen de una a seis veces por semana (42\%).

\section{Conclusiones}

- $\quad$ El $100 \%$ de la población de jóvenes universitarios de la ciudad de Armenia, participantes en este estudio comprendida entre los 18 y 25 años de edad consumen productos lácteos lo cual es reconfortante pensando en el riesgo o no de padecer osteoporosis a temprana edad.

- Se evidenció que el $48 \%$ de la población objeto de estudio consume lácteos una vez al día, el 35\% dos veces al día, el 16\% más de tres veces al día y el $1 \%$ no consume.

- En cuanto a la frecuencia de consumo, la leche en un $45 \%$ la consumen diariamente y para los demás lácteos como lo fueron el yogurt con un $52 \%$ y el queso con un $49 \% \%$ lo consumen ocasionalmente y la mantequilla la consumen de una a seis veces por semana.

- No se evidenció correlación entre el consumo, frecuencia y tipo de producto lácteo consumido vs. la cantidad de calcio encontrada en la orina y la alteración de los niveles de calcio en orina que darían origen al padecimiento de hipercalciuria o hipocalciuria.

\section{Referencias}

1. Gómez A, Paris I, Laguardia G. Osteoporosis en mujeres postmenopáusicas. [Internet]. La Habana, Cuba: Copyright 2000-2011, Revista 16 de Abril; Revista Científico Estudiantil de las Ciencias Médicas de Cuba. Diciembre de 2011. [Citado el 7 de enero de 2016]. Disponible en: http://www.16deabril.sld.cu/ rev/246/RB/3-osteoporosis\%20en\%20mujeres.html

2. Ankita M, Siris E, Xiaoqin Y, Chun Po S, Shiva S. Association Between Gastrointestinal Events and Persistence with Osteoporosis Therapy: Analysis of Administrative Claims of a U.S. Managed Care Population. J Manag Care Spec Pharm. Jun 2015;21(6):499-506.
3. González J, Del Pino J, Olmos J, Nogués X, Comisión de Redacción de las Guías de Osteoporosis de la SEIOMM. Clinical practice guidelines for postmenopausal, glucocorticoid-induced and male osteoporosis. Spanish Society for Research on Bone and Mineral Metabolism (3rd updated version 2014). Rev Clin Esp. 2015;215(9):515-526.

4. Arbonés G, Carvajal A, Gonzalvo B, González M, Joyanes M, Marques I, y cols. Nutrición y recomendaciones dietéticas para personas mayores: Grupo de trabajo „Salud pública“ de la Sociedad Española de Nutrición (SEN). [Revisión].Nutr. Hosp. 2013;18(3):109-137.

5. Carias P, Nyisztor K, Velazco G. Consumo de calcio y densidad mineral ósea en hombres jóvenes con diferentes niveles de actividad física. Rev. Venez. Endocrinol. Metab. 2014;12(1):1224.

6. Dugdale D. Calcio en Orina. [Internet]. Rockville Pike, Bethesda EE.UU: A.D.A.M., Inc. 2013. [Citado el 7 de enero de 2016]. Disponible en: https://www.nlm.nih.gov/medlineplus/spanish/ ency/article/003603.htm

7. Cardona D, Román M, Valencia F. El calcio en el desarrollo de alimentos funcionales. Rev. Lasallista Investig. 2011;8(1):104-116.

8. Schurman L, Bagur A, Claus H, Messina O, Negri A, Sánchez A, y cols. Guías 2012 para el diagnóstico, la prevención y el tratamiento de la osteoporosis. Medicina (Buenos Aires). Feb 2013;73(1):5574.

9. Bartolomé R, Forcén T, Hervás A, De Mendoza M. Manejo de osteoporosis en atención primaria. Anales Sis San Navarra. 2005;28(3):411-415.

10. Mazeto G, Da Silva F, Mangini S, Souza L. Auto-administrando el tratamiento de la osteoporosis en el rescate del bienestar, mediado por la invisibilidad de indicadores de la enfermedad. Rev. LatinoAm. Enfermagem. 2010;18(3):398-405.

11. González M, Rivera A, Morán L. Estudio nutricional para evaluar el aporte de calcio sobre la dieta de una leche fermentada enriquecida en calcio y vitamina D (Densia) en mujeres postmenopáusicas. Nutr. Hosp. Mar-Abr 2012;27(2):537-541.

12. Nyisztor J, Diamela P, Velazco Y. Consumo de calcio y densidad mineral ósea en hombres jóvenes con diferentes niveles de actividad física. Rev. Venez. Endocrinol. Metab. 2014;12(1):12-24.

13. Dugdale D. Calcio en Orina. [Internet]. Rockville Pike, Bethesda EE.UU: A.D.A.M., Inc. 2013. [Citado el 7 de enero de 2016]. Disponible en: https://www.nlm.nih.gov/medlineplus/spanish/ ency/article/003603.htm

14. González D. Hipercalciuria. Pediatr Integral. 2013;17(6):422-432. 